\title{
AN ESTIMATE FOR PSEUDOCONFORMAL CAPACITIES ON THE SPHERE
}

\author{
H.M. Reimann
}

The group $\mathrm{SU}(1, n)$ acts as a group of pseudoconformal transformations on the sphere $S=\left\{w \in \mathbf{C}^{n}:|w|=1\right\},(n \geq 2)$. Each element $g \in \mathrm{SU}(1, n)$ preserves both the contact structure given by the differential form

$$
\vartheta=i \sum_{j=1}^{n}\left(-\bar{w}_{j} d w_{j}+\omega_{j} d \bar{w}_{j}\right)
$$

and the pseudoconformal structure determined by the Leviform $L^{S}$.

There is a notion of capacity for condensers $\left(S, C_{0}, C_{1}\right)$ on the sphere, which is invariant under biholomorphic mappings (cf. [6] or [4]). If $C_{0}$ and $C_{1}$ are two disjoint non empty compact subsets of $S$, then the class of admissible functions $F=F\left(C_{0}, C_{1}\right)$ is defined as

$$
F=\left\{f \in C^{\infty}(S):\left.f\right|_{C_{0}}<0,\left.f\right|_{C_{1}}>1\right\} .
$$

The horizontal gradient $\nabla f$ of $f \in C^{\infty}(S)$ is the unique tangent vector in

$$
\mathrm{PS}=\{X \in \mathrm{TS}: \vartheta(x)=0\}
$$

such that $L^{S}(\nabla f, X)=X f$ for all $X \in \mathrm{PS}$. The capacity of the condenser is now defined by

$$
\operatorname{cap}\left(S, C_{0}, C_{1}\right)=\inf _{f \in F} \int_{S}|\nabla f|^{2 n} d \sigma
$$

where $|\nabla f|^{2}=L^{S}(\nabla f, \nabla f)$.

In particular, the capacity is invariant under the action of $\mathrm{SU}(1, n)$. In fact any $g \in \mathrm{SU}(1, n)$ is a contact transformation. Therefore

$$
g^{*} \vartheta=\lambda \vartheta
$$

and

$$
\left.g^{*} d \vartheta\right|_{\mathrm{PS}}=\left.\lambda d \vartheta\right|_{\mathrm{PS}}
$$


for some real valued function $\lambda$. The Leviform $L^{S}$ is determined by $d \vartheta$ and the complex structure $J$ of the underlying space

$$
\begin{gathered}
J \frac{\partial}{\partial x_{j}}=\frac{\partial}{\partial y_{j}} \quad J \frac{\partial}{\partial y_{j}}=-\frac{\partial}{\partial x_{j}} \quad j=1, \ldots, n \\
L^{S}(X, Y)=d \vartheta(X, J Y) \quad X, Y \in \mathrm{PS} .
\end{gathered}
$$

Since $g$ is the restriction to $S$ of a biholomorphic mapping, it follows that

$$
\begin{aligned}
L^{S}\left(g_{*} X, g_{*} Y\right) & =d \vartheta\left(g_{*} X, J g_{*} Y\right)=d \vartheta\left(g_{*} X, g_{*} J Y\right) \\
& =\left(g^{*} d \vartheta\right)(X, J Y)=\lambda d \vartheta(X, J Y)=\lambda L^{S}(X, Y)
\end{aligned}
$$

The volume element $d \sigma$ is equal to $2^{-2 n+1} \vartheta \wedge(d \vartheta)^{n-1}$ and the (real) Jacobian determinant of $g: S \rightarrow S$ is thus

$$
\operatorname{det} g_{*}=\lambda^{n} \text {. }
$$

The admissible functions for the condenser $\left(S, g C_{0}, g C_{1}\right)$ are the functions $f$ with $f \circ g \in F\left(C_{0}, C_{1}\right)$.

From the relation

$$
|\nabla(f \circ g)|=\lambda^{1 / 2}|\nabla f| \circ g
$$

it then follows that

$$
\begin{aligned}
\operatorname{cap}\left(S, g C_{0}, g C_{1}\right) & =\inf _{f \in F\left(g C_{0}, g C_{1}\right)} \int_{S}|\nabla f|^{2 n} d \sigma \\
& =\inf _{f \circ g \in F\left(C_{0}, C_{1}\right)} \int_{S}|\nabla(f \circ g)|^{2 n} \lambda^{-n} \operatorname{det} g_{*} d \sigma \\
& =\inf _{h \in F\left(C_{0}, C_{1}\right)} \int_{S}|\nabla h|^{2 n} d \sigma=\operatorname{cap}\left(S, C_{0}, C_{1}\right) .
\end{aligned}
$$

The $U(n)$-invariant distance on the sphere $S \subset \mathrm{C}^{n}$ which will be used in the sequel is

$$
d(z, w)=2^{1 / 2}|1-\langle z, w\rangle|^{1 / 2}
$$

with

$$
\langle z, w\rangle=\sum_{j=1}^{n} z_{j} \bar{w}_{j} .
$$

In particular, the diameter of a set in $S$ is defined in terms of this distance. 
Theorem. There exists a positive constant $K$ (depending on the dimension only) with the following property: If $C_{0}$ and $C_{1}$ are disjoint compact continua, each of diameter $\geq \delta$, then

$$
\operatorname{cap}\left(S, C_{0}, C_{1}\right) \geq K \delta
$$

The Cayley transform $\gamma$

$$
\begin{aligned}
z_{j} & =\frac{i w_{j}}{1+w_{n}}, \quad j=1, \ldots, n-1 \\
z_{n} & =i \frac{1-w_{n}}{1+w_{n}}
\end{aligned}
$$

maps the unit ball $B \subset \mathbf{C}^{n}$ biholomorphically onto the domain

$$
D=\left\{\left(z, z_{n}\right) \in \mathbf{C}^{n-1} \times \mathbf{C}: \operatorname{Im} z_{n}-|z|^{2}>0\right\} .
$$

It is a pseudoconformal mapping of $S \backslash\left\{-e_{n}\right\}$ onto $\delta D$. This boundary is a realisation of the Heisenberg group $H=H^{n-1}(n \geq 2)$. Expressed in the coordinates $(z, t) \in \mathbf{C}^{n-1} \times \mathbf{R}\left(t=\operatorname{Re} z_{n}\right)$ the contact structure is determined by the differential form

$$
\omega=i \sum_{j=1}^{n-1}\left(-\bar{z}_{j} d z_{j}+z_{j} d \bar{z}_{j}\right)+d t
$$

and the Leviform on $\mathrm{PH}=\{x \in \mathrm{TH}: \omega(x)=0\}$ is

$$
L^{H}(X, Y)=d \omega(X, J Y) \text {. }
$$

The Cayley transform $\gamma$, restricted to $S \backslash\left\{-e_{n}\right\}$ satisfies

$$
\begin{gathered}
\gamma^{*} \omega=\frac{1}{\left|1+w_{n}\right|^{2}} \vartheta \\
L^{H}\left(\gamma_{*} X, \gamma_{*} Y\right)=d \omega\left(\gamma_{*} X, J \gamma_{*} Y\right)=\frac{1}{\left|1+w_{n}\right|^{2}} L^{S}(X, Y) .
\end{gathered}
$$

The pseudoconformal capacity for a condenser $\left(H, C_{0}, C_{1}\right)$ on the Heisenberg group can now be defined as before:

$$
\operatorname{cap}\left(H, C_{0}, C_{1}\right)=\inf _{f \in F} \int_{H}|\nabla f|^{2 n} d v
$$

with

$$
d v=d x_{1} \wedge d y_{1} \wedge \cdots \wedge d y_{n} \wedge d t=2^{-2 n+2} \omega \wedge(d \omega)^{n-1}
$$




$$
F=\left\{f \in C^{\infty}(H):\left.f\right|_{C_{0}}<0,\left.f\right|_{C_{1}}>1\right\} .
$$

The norm of the horizontal gradient $\nabla f$ is of course defined with respect to the Leviform $L^{H}$.

Since the Cayley transform $\gamma$ is a pseudoconformal transformation $\gamma$ : $S \backslash\left\{-e_{n}\right\} \rightarrow H$, it follows that

$$
\operatorname{cap}\left(H, \gamma C_{0}, \gamma C_{1}\right)=2 \operatorname{cap}\left(S, C_{0}, C_{1}\right) .
$$

The factor 2 has its origin in the volume relations

$$
\gamma^{*} d v=2^{-2 n+2} \gamma^{*} \omega \wedge\left(\gamma^{*} d \omega\right)^{n-1}=2^{-2 n+2} \lambda^{n} \vartheta \wedge(d \vartheta)^{n-1}=2 \lambda^{n} d \sigma .
$$

In addition to the preceding considerations, the proof requires a standard approximation argument, showing that functions $f$ in $C^{\infty}\left(S \backslash\left\{-e_{n}\right\}\right)$ with $\int_{S}|\nabla f|^{2 n}$ $<\infty$ can be suitably approximated by functions in $C^{\infty}(S)$. With these observations, the theorem can be reformulated for the Heisenberg group. The following corollary is then a special case.

Corollary. If $C_{0}$ and $C_{1}$ are closed disjoint continua, if $C_{0}$ connects the origin to the unit sphere

$$
U=\left\{(z, t) \in H:\|(z, t)\|=\left(|z|^{4}+t^{2}\right)^{1 / 4}=1\right\}
$$

and if $C_{1}$ connects the unit sphere to the point $\infty$ (i.e. $C_{1}$ is unbounded), then

$$
\operatorname{cap}\left(H, C_{0}, C_{1}\right) \geq 2 K \sqrt{2} .
$$

For the proof, observe that $U$ is the image of the sphere $E=\{w \in S$ : $\left.\operatorname{Re} w_{n}=0\right\}$ under the Cayley transform $\gamma$. Therefore, $\operatorname{diam} \gamma^{-i} C_{j} \geq \sqrt{2}$ for $j=1,2$.

For the theory of quasiconformal mappings on the Heisenberg group, this estimate is crucial in establishing the passage from the "analytic" to the "metric" definition of quasiconformality. Also, local Hölder estimates and various results on normal families of quasiconformal mappings are intimately related to this inequality. The corresponding capacity estimate for rings in $\mathbf{R}^{n}$ is based on symmetrization (Gehring [2]) or on modulus estimates for curve families on hypersurfaces (Väisälä [9]). So far, neither of these methods could be transferred to the analysis on the Heisenberg group. On the other hand, the proof of the theorem, which is stated here for the Heisenberg group, simplifies on $\mathbf{R}^{n}$. It provides a direct access to the basic capacity estimate for the theory of quasiconformal mappings in $\mathbf{R}^{n}$.

The remainder of this paper contains the complete proof for the theorem. Various auxiliary results, which are of an elementary nature, are assembled in the following lemmata. 
Lemma 1. If $f \in C^{\infty}(S)$ then

$$
\left|f\left(e_{n}\right)-f\left(-e_{n}\right)\right| \leq(n-1) a_{n-1} \int_{S}|\nabla f|(\omega)\left(\frac{2}{d\left(w, e_{n}\right) d\left(w,-e_{n}\right)}\right)^{2 n-1} d \sigma
$$

with $a_{n-1}=2^{n-4} \pi^{-n}\left(\Gamma\left(\frac{1}{2}(n-1)\right)\right)^{2}$.

For the proof, consider a function $u \in C^{\infty}\left(H^{n-1}\right)$ with compact support. The fundamental solution of the sublaplacian

$$
L=-\frac{1}{4} \operatorname{div} \nabla=-\frac{1}{2} \sum_{j=1}^{n-1}\left(\bar{Z}_{j} Z_{j}+Z_{j} \bar{Z}_{j}\right)
$$

is given by

$$
g=\|(z, t)\|^{-2 n+2} a_{n-1}, \quad a_{n}=2^{n-2} \frac{\Gamma^{2}\left(\frac{1}{2} n\right)}{\pi^{n-1}}
$$

(see Folland-Stein [1]). Therefore

$$
u(0)=\int_{H^{n-1}} g L u d v=\frac{1}{4} a_{n-1} \int_{H^{n-1}}(\nabla u, \nabla g) d v .
$$

If one uses the fact that $([4, \mathrm{p} .15])$

this leads to the estimate

$$
|\nabla\|(z, t)\||=\frac{|z|}{\|(z, t)\|}
$$

$$
|u(0)| \leq \frac{1}{4} a_{n-1} \int_{H^{n-1}}|\nabla u|(2 n-2)\|(z, t)\|^{-2 n+1} d v .
$$

The Cayley transform permits the transfer of this inequality to the sphere. Set $f=u \circ \gamma$ and observe that $f\left(-e_{n}\right)=0$. From the relations

$$
\begin{gathered}
\|(z, t)\|=\frac{d\left(w, e_{n}\right)}{d\left(w,-e_{n}\right)} \quad \text { if } \quad(z, t)=\gamma(\omega), \\
\gamma^{*} \omega=\lambda \vartheta, \quad \lambda=4 d\left(w, e_{n}\right)^{-4}, \\
|\nabla u|=\lambda^{1 / 2}|\nabla f| \\
d v=2 \lambda^{n} d \sigma
\end{gathered}
$$

it then follows that

$$
\begin{aligned}
\left|f\left(e_{n}\right)-f\left(-e_{n}\right)\right| & \leq(n-1) a_{n-1} \int_{\Sigma}|\nabla f| \lambda^{-1 / 2}\left(\frac{d\left(w, e_{n}\right)}{d\left(w,-e_{n}\right)}\right)^{-2 n+1} \lambda^{n} d \sigma \\
& =(n-1) a_{n-1} \int_{\Sigma}|\nabla f|\left(\frac{2}{d\left(w, e_{n}\right) d\left(w,-e_{n}\right)}\right)^{2 n-1} d \sigma .
\end{aligned}
$$

This proves the lemma for functions $f \in C^{\infty}(S)$ with $f=0$ in a neighbourhood of $-e_{n}$. The general case follows by approximation. 
Lemma 2. For any $f \in C^{\infty}(S)$ we have

$$
|f(x)-f(y)| \leq(n-1) a_{n-1} 2^{2 n-1} \int_{S}|\nabla f(z)|\left(d(x, z)^{-2 n+1}+d(y, z)^{-2 n+1}\right) d \sigma(z) .
$$

For fixed $x, y \in S$ choose an automorphism $g \in \mathrm{SU}(1, n)$ with $g(x)=e_{n}$, $g(y)=-e_{n}, g(z)=w$ and set $a=g^{-1}(0)$. The following identities will be applied (see Rudin [8, p. 28]).

$$
\begin{aligned}
1-\langle g(z), g(x)\rangle & =\frac{(1-\langle a, a\rangle)(1-\langle z, x\rangle)}{(1-\langle z, a\rangle)(1-\langle a, x\rangle)}, \\
\lambda(z) & =\frac{1-\langle a, a\rangle}{|1-\langle z, a\rangle|^{2}} .
\end{aligned}
$$

It follows that

$$
\begin{aligned}
\frac{1}{\left|1-\left\langle w, e_{n}\right\rangle\right|\left|1-\left\langle w,-e_{n}\right\rangle\right|} & =\lambda^{-1}(z) \frac{|1-\langle a, x\rangle||1-\langle a, y\rangle|}{|1-\langle a, a\rangle||1-\langle z, x\rangle||1-\langle z, y\rangle|} \\
& =\frac{1}{2} \lambda^{-1}(z) \frac{1-\langle x, y\rangle}{|1-\langle z, x\rangle||1-\langle z, y\rangle|} .
\end{aligned}
$$

The change of variables $w=g(z)$ in the integral of Lemma 1 then gives

$$
\begin{aligned}
\mid(f \circ g)(x) & -(f \circ g)(y)|=| f\left(e_{n}\right)-f\left(-e_{n}\right) \mid \\
& =(n-1) a_{n-1} \int_{S}|\nabla(f \circ g)(z)|\left(\frac{d(x, y)}{d(z, x) d(z, y)}\right)^{2 n-1} d \sigma(z) .
\end{aligned}
$$

Together with the relation

$$
\begin{aligned}
\left(\frac{d(x, y)}{d(z, x) d(z, y)}\right)^{2 n-1} & \leq\left(\frac{1}{d(z, x)}+\frac{1}{d(z, y)}\right)^{2 n-1} \\
& \leq 2^{2 n-1}\left(d(z, x)^{-2 n+1}+d(z, y)^{-2 n+1}\right)
\end{aligned}
$$

this shows that

$$
|(f \circ g)(x)-(f \circ g)(y)| \leq K \int_{S}|\nabla(f \circ g)(z)|\left(d(z, x)^{-2 n+1}+d(z, y)^{-2 n+1}\right) d \sigma(z)
$$

with $K=(n-1) a_{n-1} 2^{2 n-1}$.

For the following consideration a volume estimate for the balls

$$
B(x, r)=\{y \in S: d(x, y)<r\} \subset S
$$


is required. From the equation

$$
\|(z, t)\|=\frac{d\left(\omega, e_{n}\right)}{d\left(\omega,-e_{n}\right)}, \quad(z, t)=\gamma(\omega),
$$

it can be concluded that

$$
\left|B\left(e_{n}, r\right)\right|=\int_{d\left(w, e_{n}\right)<r} d \sigma(w)
$$

is of the order $r^{2 n}$ for small $r$. There exists a constant $c$ such that

$$
c^{-1} r^{2 n} \leq|B(x, r)| \leq c r^{2 n}, \quad r \in(0,2] .
$$

The integral in Lemma 2 can then be evaluated in the form

$$
\int_{S}|\nabla f(z)| d(x, z)^{-2 n+1} d \sigma(z)=\int_{0}^{2} r^{-2 n+1}\left(\int_{d(x, z)=r}|\nabla f(z)| d \varrho\right) d r
$$

where $d \sigma=d \varrho d r$. Partial integration leads to the inequality

$$
\begin{aligned}
& \int_{S}|\nabla f(z)| d(x, z)^{-2 n+1} d \sigma(z) \\
& \quad \leq(2 n-1) \int_{0}^{2} r^{-2 n}\left(\int_{B(x, r)}|\nabla f(z)| d \sigma(z)\right) d r+2^{-2 n+1} \int_{S}|\nabla f(z)| d \sigma(z) .
\end{aligned}
$$

Lemma 3. For fixed $f \in C^{\infty}(S)$ and for any $\alpha>0$ set

$$
F_{\alpha}=\left\{x \in S: \int_{B(x, r)}|\nabla f|^{2 n} d \sigma<\alpha^{-2 n} r \quad \text { for all } r \in(0,2]\right\} \text {. }
$$

There exist constants $K_{1}, K_{2}$ depending on $n$ only such that

$$
|f(x)-f(y)| \leq \frac{K_{1}}{\alpha}+K_{2}\|\nabla f\|_{2 n}
$$

for all $x, y \in F_{\alpha}$.

Hölder's inequality and the vulume estimate give the inequality

$$
\int_{B(x, r)}|\nabla f| d \sigma \leq c^{1-(1 / 2 n)} r^{2 n-1}\left(\int_{B(x, r)}|\nabla f|^{2 n} d \sigma\right)^{1 / 2 n} .
$$


With the assumption $x \in F_{\alpha}$, this implies

$$
\int_{B(x, r)}|\nabla f| d \sigma \leq c^{1-(1 / 2 n)} r^{2 n-1+(1 / 2 n)} \alpha^{-1} .
$$

The previous evaluation of the integral in Lemma 2 then shows that

$$
\begin{aligned}
& |f(x)-f(y)| \\
& \leq(2 n-1) \int_{0}^{2} r^{-2 n}\left(\int_{B(x, r)}|\nabla f| d \sigma+\int_{B(y, r)}|\nabla f| d \sigma\right) d r+2^{-2 n+2} \int_{S}|\nabla f| d \sigma \\
& \leq 2(2 n-1) \int_{0}^{2} c^{1-(1 / 2 n)} r^{-1+(1 / 2 n)} \alpha^{-1} d r \\
& \quad+2^{-2 n+2}\left(\int_{S} d \sigma\right)^{1-(1 / 2 n)}\left(\int_{S}|\nabla f|^{2 n} d \sigma\right)^{1 / 2 n} \\
& =K_{1} \alpha^{-1}+K_{2}\|\nabla f\|_{2 n}
\end{aligned}
$$

provided that $x, y \in F_{\alpha}$.

The following covering lemma is taken from Korányi-Vági [3].

Lemma 4. Given any function $r: A \rightarrow(0,2]$ defined on a subset $A \subset S$, there exists a (finite or infinite) sequence $\left\{x_{n}\right\} \subset A$ such that the balls $B\left(x_{n}, r\left(x_{n}\right)\right) \subset S$ are mutually disjoint and such that

$$
A \subset \bigcup_{n} B\left(x_{n}, 3 r\left(x_{n}\right)\right) .
$$

The approximating linear Hausdorff measure of a set $A \subset S$ is defined by

$$
\Lambda(A)=\inf \left\{\sum_{n} r_{n}: A \subset \bigcup_{n} B\left(x_{n}, r_{n}\right)\right\} .
$$

The covering lemma shows that

$$
\Lambda\left(S \backslash F_{\alpha}\right) \leq 3 \alpha^{2 n}\|\nabla f\|_{2 n}^{2 n}
$$

if $F_{\alpha}$ is the set defined in Lemma 3. In fact, for any point $x \in S \backslash F_{\alpha}$ there exists $r(x)$ with

$$
\int_{B(x, r(x))}|\nabla f|^{2 n} \geq \alpha^{-2 n} r(x) .
$$

Choose mutually disjoint balls $B\left(\left(x_{j}, r\left(x_{j}\right)\right)\right.$ with

$$
\left(S \backslash F_{\alpha}\right) \subset \bigcup_{j} B\left(x_{j}, 3 r\left(x_{j}\right)\right)
$$


then

$$
\Lambda\left(S \backslash F_{\alpha}\right) \leq \sum_{j} 3 r\left(x_{j}\right) \leq 3 \alpha^{2 n} \sum_{j} \int_{B\left(x_{j}, r\left(x_{j}\right)\right)}|\nabla f|^{2 n} d \sigma \leq 3 \alpha^{2 n} \int_{S}|\nabla f|^{2 n} d \sigma .
$$

With these preliminaries, the main result can now easily be proved. Given a condenser $\left(S, C_{0}, C_{1}\right)$ consisting of disjoint compact sets $C_{j} \subset S, j=1,2$ and given $\varepsilon>0$, there exists an admissible function $f \in F\left(C_{0}, C_{1}\right)$ with

$$
\operatorname{cap}\left(S, C_{0}, C_{1}\right) \geq\|\nabla f\|_{2 n}^{2 n}-\varepsilon
$$

If the inequality $\|\nabla f\|_{2 n} \geq\left(2 K_{2}\right)^{-1}$ holds, then

$$
\operatorname{cap}\left(S, C_{0}, C_{1}\right) \geq\left(2 K_{2}\right)^{-2 n}-\varepsilon .
$$

Otherwise Lemma 3 will be applied with $\alpha=2 K_{1}$, showing that

$$
|f(x)-f(y)| \leq \frac{K_{1}}{\alpha}+K_{2}\|\nabla f\|_{2 n}<1
$$

for all $x, y \in F_{\alpha}$. Since $f$ is admissible, at most one of the sets $C_{j}$ can contain points of $F_{\alpha}$

$$
C_{j} \cap F_{\alpha}=\emptyset \quad \text { for } j=0 \text { or } j=1 \text {. }
$$

But this implies in view of the covering lemma

$$
\begin{aligned}
& \Lambda\left(C_{j}\right) \leq \Lambda\left(S \backslash F_{\alpha}\right) \leq 3 \alpha^{2 n}\|\nabla f\|_{2 n}^{2 n}, \\
& \Lambda\left(C_{j}\right) \leq 3\left(2 K_{1}\right)^{2 n}\left(\operatorname{cap}\left(S, C_{0}, C_{1}\right)+\varepsilon\right) .
\end{aligned}
$$

In any case, this shows that the smallest of the numbers

$$
\left(2 K_{2}\right)^{-2 n}, \quad 3^{-1}\left(2 K_{1}\right)^{-2 n} \Lambda\left(C_{0}\right), \quad 3^{-1}\left(2 K_{1}\right)^{-2 n} \Lambda\left(C_{1}\right)
$$

is a lower bound for $\operatorname{cap}\left(S, C_{0}, C_{1}\right)$ and establishes the existence of a positive constant $K$ such that

$$
\operatorname{cap}\left(S, C_{0}, C_{1}\right) \geq K \min _{j=1,2}\left\{\Lambda\left(C_{j}\right)\right\}
$$

The observation that $\operatorname{diam} C_{j} \leq 2 \Lambda\left(C_{j}\right)$ for any compact continuum $C_{j}$ then completes the proof of the theorem.

Remark. The method used in deriving this capacity estimate goes back to Rešhetnjak [7]. As has been shown by Martio [5], various generalizations can be obtained, if approximating Hausdorff measures of any dimension are considered. 


\section{References}

[1] Folland, G.B., and E.M. Stein: Estimates for the $\bar{\partial}_{b}$ complex and analysis on the Heisenberg group. - Comm. Pure Appl. Math. 27, 1974, 429-522.

[2] Gehring, F.W.: Symmetrization of rings in space. - Trans. Amer. Math. Soc. 101, 1961, 499-519.

[3] KorÁNYI, A., and S. VÁGI: Singular integrals on homogeneous spaces and some problems of classical analysis. - Ann. Scuola Norm. Sup. Pisa Cl. Sci. (4) 25, 1971, 575-648.

[4] KorÁNYI, A., and H.M. REIMANN: Horizontal normal vectors and conformal capacity of spherical rings in the Heisenberg group. - Bull. Sci. Math. (2) 111, 1987, 3-21.

[5] Martio, O.: Capacity and measure densities. - Ann. Acad. Sci. Fenn. Ser. A I Math. 4, 1978/79, 109-118.

[6] Pansu, P.: Métrique de Carnot-Carathéodory et quasiisométries des espaces symétriques de rang un. - To appear.

[7] REŠHetNJAK, JU.G.: The concept of capacity in the theory of functions with generalized derivatives. - Sibirsk. Mat. Z̆. 10, 1969, 1109-1138 (Russian).

[8] Rudin, W.: Function theory in the unit ball of $\mathbf{C}^{n}$. - Grundlehren der Mathematik 241. Springer-Verlag, New York-Heidelberg-Berlin, 1980.

[9] VÄIs̈̈LÄ, J.: Lectures on $n$-dimensional conformal mappings. - Lecture Notes in Mathematics 229. Springer-Verlag, Berlin-Heidelberg-New York, 1971.

Universität Bern

Mathematisches Institut

Sidlerstrasse 5

CH-3012 Bern

Schweiz

Received 19 September 1988 